15

\title{
Методика определения удельного сопротивления полупроводниковых материалов методом атомно-силовой микроскопии
}

\author{
() В.А. Смирнов, ${ }^{1}$ Р.В. Томинов, ${ }^{1}$ Н.И. Алябьева, ${ }^{2}$ М.В. Ильина, ${ }^{1}$ В.В. Полякова, ${ }^{1}$ Ал.В. Быков, ${ }^{1}$ О.А. Агеев ${ }^{1}$ \\ ${ }^{1}$ Южный федеральный университет, Институт нанотехнологий, электроники и приборостроения, \\ 347922 Таганрог, Россия \\ ${ }^{2}$ University of Paris-Sud, Orsay cedex, France \\ e-mail: vasmirnov@sfedu.ru
}

(Поступило в Редакцию 24 мая 2017 г.)

Показаны результаты теоретических и экспериментальных исследований поверхности подложек кремния методом атомно-силовой микроскопии в режиме отображения сопротивления растекания, а также представлены разработки методики определения удельного сопротивления полупроводниковых материалов на основе этих исследований. Показано наличие порогового значения силы прижима зонда к поверхности подложки, при превышении которого удельное сопротивление кремния определяется достоверно. Исследовано влияние окружающей среды на значения токов в системе зонд-подложка. Показано, что для получения достоверных результатов исследования электрических параметров полупроводниковых материалов методом атомносиловой микроскопии в режиме отображения сопротивления растекания необходимо проводить в условиях сверхвысокого вакуума.

DOI: 10.21883/JTF.2018.08.46320.2351

\section{Введение}

Одним из основных условий получения качественного результата при изготовлении современных приборов наноэлектроники является наличие высокоточных инструментов диагностики формируемых наноструктур. Зачастую при переходе к технологическому процессу с более высокими проектно-топологическими нормами ранее использовавшиеся инструменты диагностики оказываются неприменимыми из-за достижения предела их разрешающей способности. Поэтому возникает необходимость в разработке более точных методов исследования электрофизических свойств материалов формируемых наноструктур, которые обладали бы достаточно высоким пространственным разрешением, высокой степенью достоверности и воспроизводимости [1-11].

Атомно-силовая микроскопия в режиме отображения сопротивления растекания (АСМ ОСР) является многофункциональным методом зондовой нанодиагностики, который позволяет проводить исследование распределения тока по поверхности образца с одновременной визуализацией ее топологии [12]. Данный метод применяется для исследования электрофизических параметров различных наноразмерных структур: графенов $[1,6]$, углеродных нанотрубок [2], нитевидных нанокристаллов $\mathrm{ZnO}$ [3], эпитаксиальных наногетероструктур [4,5]. Однако при проведении исследований материалов методом АСМ ОСР возникает ряд проблем, связанных, во-первых, с отсутствием методик, позволяющих определять электрические параметры исследуемых материалов из полученных результатов, во-вторых, с выбором оптимальных режимов взаимодействия острия зонда с поверхностью образца, в-третьих, с недостаточной изученностью влияния параметров окружающей среды [12].
Целью работы является исследование влияния окружающей среды и силы прижима зонда к поверхности образца на удельное сопротивление подложек кремния при исследовании методом АСМ ОСР, а также разработка на основе этого исследования методики определения удельного сопротивления полупроводниковых материалов.

\section{Методика эксперимента}

Для проведения экспериментальных исследований удельного сопротивления полупроводниковых материалов методом АСМ ОСР были использованы зондыкантилеверы марки NSG11 с проводящим покрытием $\mathrm{W}_{2} \mathrm{C}$ и подложки кремния c различными значениями удельного сопротивления согласно спецификации (см. таблицу).

Перед измерениями образцы кремния были очищены в ацетоне и изопропиловом спирте при температурах 56 и $83^{\circ} \mathrm{C}$ соответственно, в течение $10 \mathrm{~min}$, а также обработаны в водном растворе НF $(10 \%)$ в течение $30 \mathrm{~s}$.

Исследование влияния окружающей среды проводилось на кремниевой подложке КДБ-10 методом АСМ ОСР при атмосферном давлении (зондовая нанолаборатория (ЗНЛ) Ntegra, НТ-МДТ, Россия) и в сверхвысоком вакууме $\left(10^{-8} \mathrm{~Pa}\right)$ (модуль сверхвысоковакуумной сканирующей зондовой микроскопии нанотехнологического комплекса НАНОФАБ НТК-9, НТ-МДТ, Россия).

Схематическое изображение определения электрических параметров полупроводниковых материалов методом АСМ ОСР представлено на рис. 1. Зонд подведен к поверхности образца в контактном режиме и воздействует на поверхность подложки кремния с силой $F_{\text {th }}$. Между зондом и подложкой подается внешнее напряжение смещения. Для определения значений токов 
Электрофизические параметры кремниевых образцов

\begin{tabular}{|c|c|c|c|c|c|c|}
\hline $\begin{array}{c}\text { Маркировка } \\
\text { образца }\end{array}$ & $\begin{array}{c}\text { Удельное } \\
\text { сопротивление } \\
\text { (спецификация), } \\
\Omega \cdot \mathrm{cm}\end{array}$ & $\begin{array}{l}\text { Тип } \\
\text { прово- } \\
\text { димо- } \\
\text { сти }\end{array}$ & $\begin{array}{l}\text { Толщина } \\
\text { образца, } \\
\mu \mathrm{m}\end{array}$ & $\begin{array}{c}\text { Сила } \\
\text { прижима } \\
\text { зонда, } \\
\mu \mathrm{N}\end{array}$ & $\begin{array}{c}\text { Экспериментальные } \\
\text { значение тока } \\
\text { растекания, } \\
\text { nA }\end{array}$ & $\begin{array}{c}\text { Удельное } \\
\text { сопротивление } \\
\text { (эксперимент), } \\
\Omega \cdot \mathrm{cm}\end{array}$ \\
\hline КЭС-0.01 & 0.01 & $n$ & \multirow{4}{*}{460} & \multirow{4}{*}{2} & $49.80 \pm 1.08$ & $0.04 \pm 0.01$ \\
\hline КДБ-1 & 1 & $p$ & & & $24.89 \pm 0.43$ & $0.70 \pm 0.10$ \\
\hline КДБ-5 & 5 & $p$ & & & $8.11 \pm 0.24$ & $3.73 \pm 0.40$ \\
\hline КДБ-10 & 10 & $p$ & & & $2.00 \pm 0.38$ & $11.24 \pm 1.20$ \\
\hline
\end{tabular}

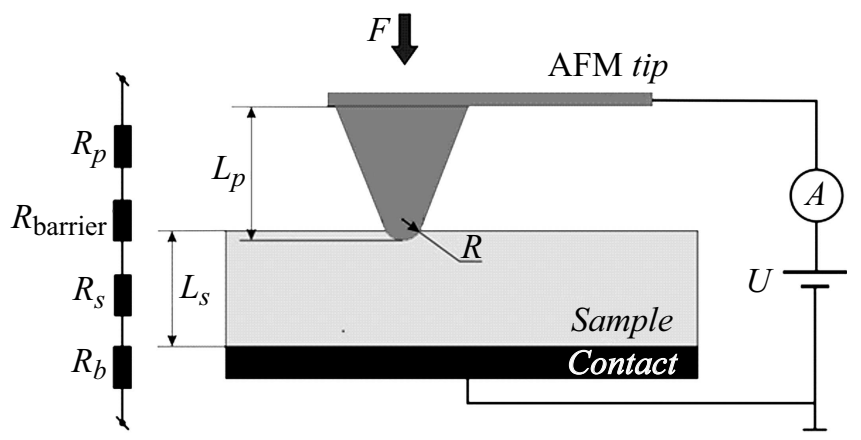

Рис. 1. Схематическое изображение определения электрических параметров полупроводниковых материалов методом ACM OCP.

в системе зонд-образец был использован встроенный в ЗНЛ осциллограф.

На первом этапе было проведено сканирование области $5 \times 5 \mu \mathrm{m}$ при атмосферном давлении в контактном режиме при подаче постоянного напряжения $3 \mathrm{~V}$ и силе прижима зонда к поверхности образца $2.5 \mu \mathrm{N}$, затем в режиме АCM ОСР была получена карта отображения тока растекания области размером $7 \times 7 \mu \mathrm{m}$ (рис. $2, a)$. На втором этапе данный эксперимент был повторно проведен в условиях сверхвысокого вакуума при тех же режимах (рис. 2,b).

Исследование влияния силы прижима зонда к поверхности образца на значения тока растекания осуществлялось на кремниевой подложке КЭС-0.01 в условиях сверхвысокого вакуума $\left(10^{-8} \mathrm{~Pa}\right)$. Сканирование проводилось при подаче постоянного напряжения $3 \mathrm{~V}$, сила прижима зонда к поверхности подложки изменялась от 0.3 до $6 \mu \mathrm{N}$. На рис. 3 представлены АСМ изображения поверхности кремния, карта отображения тока растекания и профилограмма тока по поверхности образца КЭС-0.01 при силе прижима зонда $0.3,1$ и $2 \mu \mathrm{N}$. На рис. 4 представлены семейства ВАХ, полученные на подложках кремния КЭС-0.01 и КДБ-1 методом токовой спектроскопии, при значениях силы прижима зонда 0.3, 1 и $2 \mu \mathrm{N}$. Полученные экспериментальные значения токов в системе зонд-образец использовались при раз- работке математической модели определения удельного сопротивления полупроводниковых материалов.

\section{Описание математической модели}

Для разработки методики определения удельного сопротивления полупроводниковых материалов методом АСМ ОСР была разработана математическая модель, учитывающая вклад компонентов сопротивления $\left(R_{\text {tot }}\right)$ в условиях сверхвысокого вакуума (рис. 1) [5]:

$$
R_{\mathrm{tot}}=R_{\text {barrier }}+R_{s}+R_{p}+R_{b},
$$

где $R_{\text {barrier }}$ - сопротивление контакта острия зонда с поверхностью образца, $R_{s}$ - сопротивление исследуемого образца, $R_{p}$ - сопротивление проводящего покрытия зонда, $R_{b}$ - сопротивление нижнего металлического контакта $[4,5,12]$.

Сопротивления зонда $R_{p}$ и образца $R_{s}$ определяются выражениями [5]

$$
\begin{gathered}
R_{p}=\frac{\rho_{p}}{\pi} \int_{0}^{L_{p}} \frac{d l}{\left(r_{1}+l\left(r_{2}-r_{1}\right) / L_{p}\right)^{2}-\left(r_{3}+l\left(r_{4}-r_{3}\right) / L_{p}\right)^{2}}, \\
R_{s}=\rho_{s} \frac{L_{s}}{A_{s}},
\end{gathered}
$$

где $\rho_{p}$ и $\rho_{s}$ - удельные сопротивления проводящего покрытия зонда и образца соответственно, $L_{p}$ - высота зонда, $r_{1}$ - радиус острия зонда с учетом проводящего слоя, $r_{2}$ - радиус основания зонда с учетом проводящего слоя, $r_{3}$ - радиус острия зонда без учета проводящего слоя, $r_{4}$ - радиус основания зонда без учета проводящего слоя, $L_{s}$ - толщина исследуемого образца, $A_{s}$ - площадь контакта зонд-образец.

Значение сопротивления контакта острия зонда с поверхностью подложки определяется уравнением Шарвина $[5,12]$

$$
R_{\text {barrier }}=\frac{\rho}{\pi a^{2}}, \quad \rho=\frac{\rho_{p} \lambda_{p}+\rho_{s} \lambda_{s}}{2},
$$

где $\lambda_{p}, \lambda_{s}$ - длина свободного пробега носителей заряда в проводящем покрытии зонда и подложке соответственно, $\rho$ - переходное контактное сопротивление зонда и 

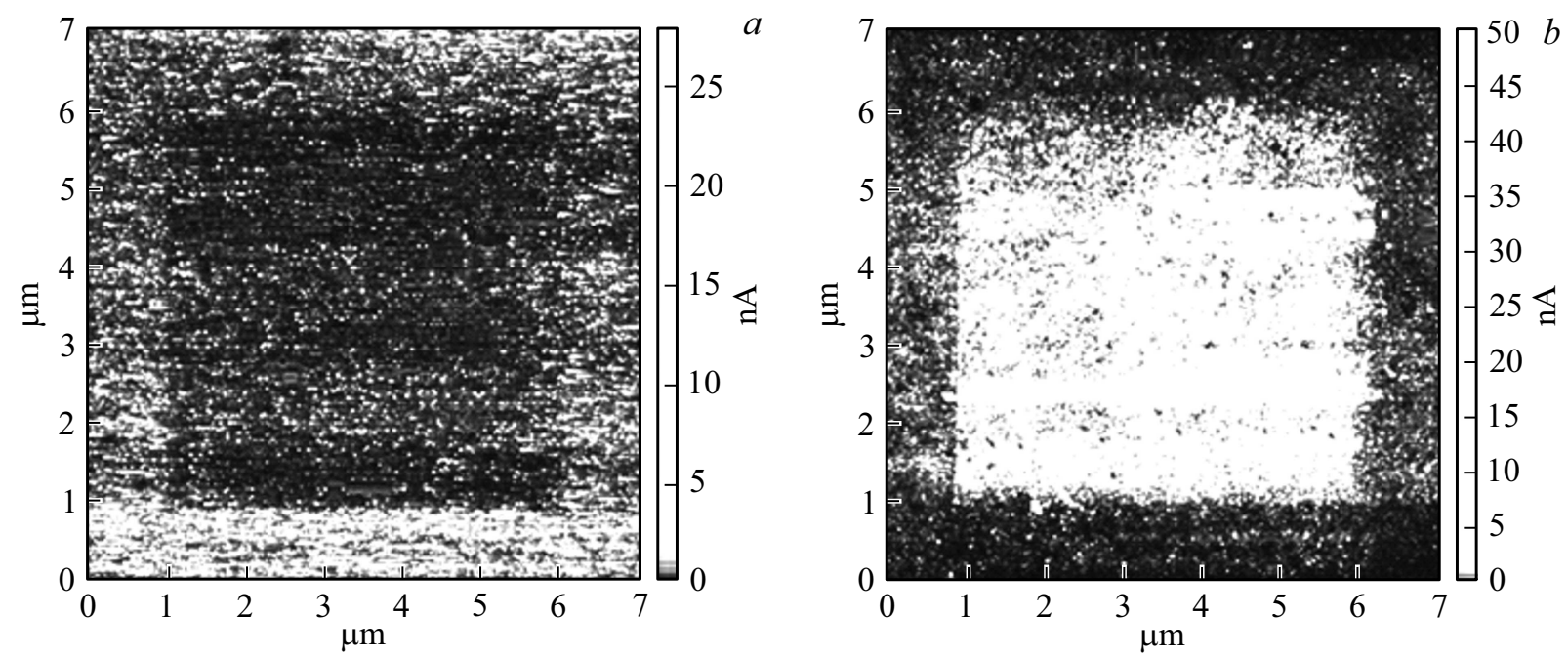

Рис. 2. АСМ изображения распределения тока растекания по поверхности кремниевой подложки марки КДБ-10, полученные: $a-$ при атмосферном давлении, $b-$ в сверхвысоком вакууме $\left(10^{-8} \mathrm{~Pa}\right)$.

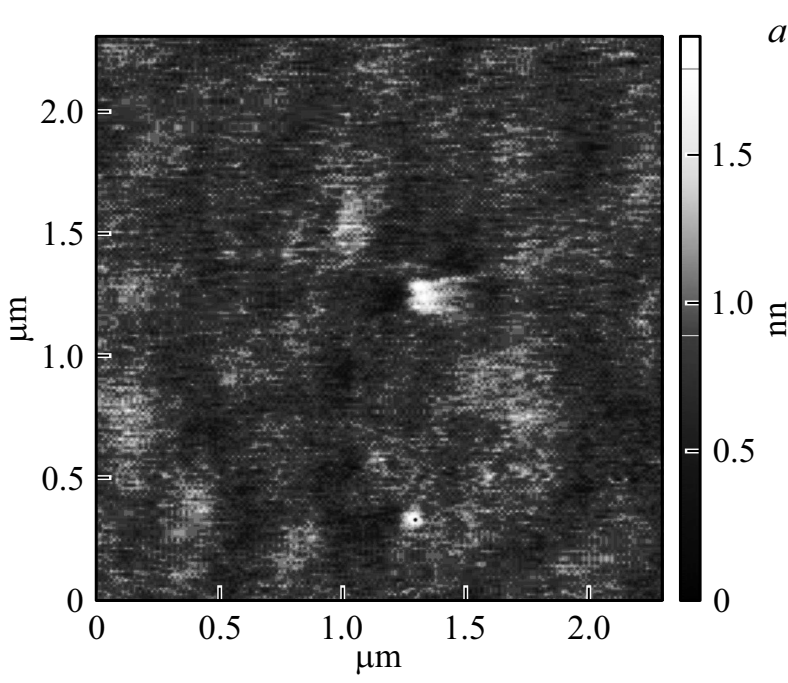

Weak

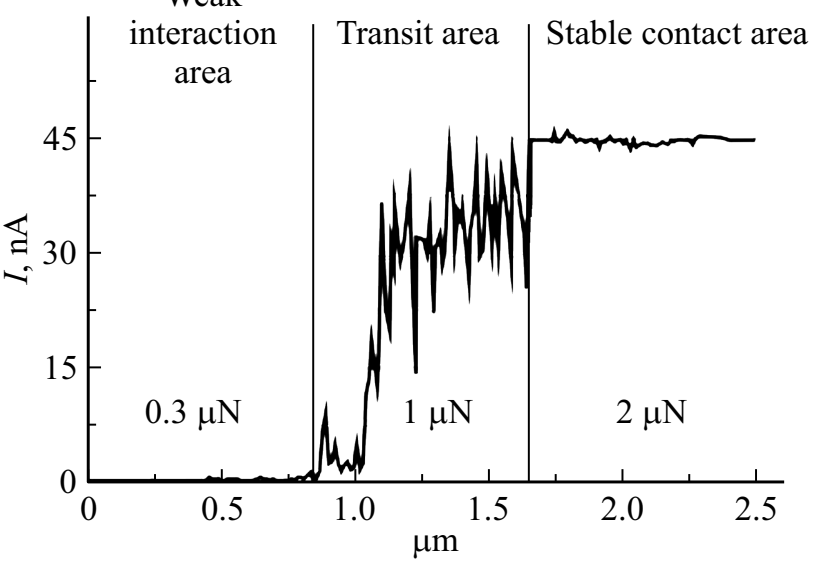

Рис. 3. АСМ изображения поверхности кремниевой подложки КЭС-0.01, полученные при различных силах прижима зонда к поверхности образца: $a-$ морфология поверхности, $b-$ отображение сопротивления растекания, $c-$ профилограмма тока вдоль линии на $b$. 


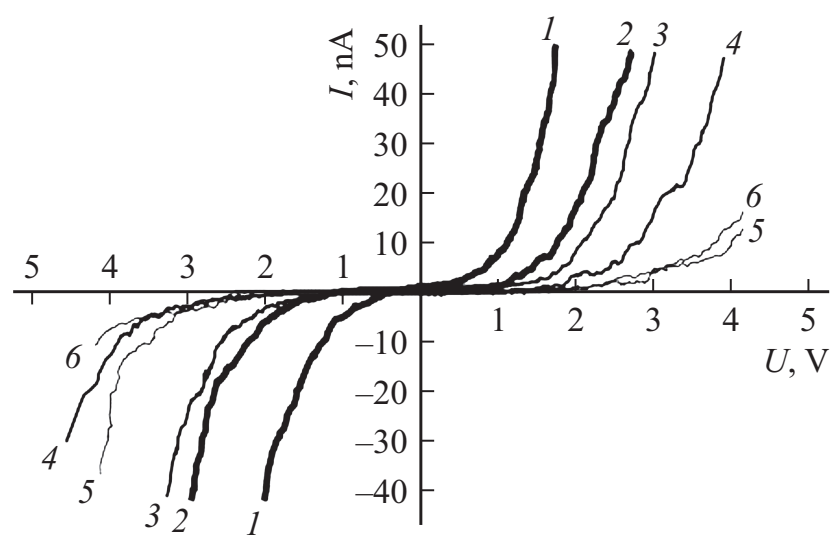

Рис. 4. Вольт-амперные характеристики для кремниевых подложек КЭС-0.01 $(1,3,5)$ и КДБ-1 $(2,4,6)$ при различных силах прижима зонда к поверхности образца: $1,2-2,3,4-1$, $5,6-0.3 \mu \mathrm{N}$.

подложки, $a$ - радиус контакта зонда с поверхностью образца, зависящий от силы прижима зонда к поверхности подложки, определяется согласно модели Герца [13]

$$
a=\sqrt[3]{\frac{F_{\mathrm{th}} r_{1}}{E^{*}}}
$$

где $F_{\text {th }}$ - сила прижима зонда к поверхности образца, $E^{*}$ - приведенный модуль Юнга материалов системы зонд-образец [11].

$$
\frac{1}{E^{*}}=\frac{3}{4}\left(\frac{1-v_{p}^{2}}{E_{p}}+\frac{1-v_{s}^{2}}{E_{s}}\right)
$$

где $v_{p}, v_{s}-$ коэффициенты Пуассона материалов острия зонда и образца, $E_{p}, E_{s}$ - модули Юнга материалов острия зонда и образца.
С использованием (1)-(5) было получено выражение, позволяющее определить удельное сопротивление материала исследуемого образца в условиях сверхвысокого вакуума

$$
\rho_{s}=\left[\frac{U}{I} \mathrm{CF}-R_{b}-\rho_{p}\left(\frac{2 \lambda_{p}}{3 \pi a^{2}}+\frac{L_{p}}{A_{p}}\right)\right] /\left(\frac{2 \lambda_{s}}{3 \pi a^{2}}+\frac{L_{s}}{A_{s}}\right),
$$

где CF - корректирующий фактор. Введение CF обосновано проявлением различных эффектов в приконтактной области, которые оказывают влияние на токопрохождение и не учитываются в рамках предложенной модели: генерационно-рекомбинационные токи на поверхностных состояниях; наличие приповерхностной области с высоким уровнем механических напряжений, приводящих к смещению энергетических уровней разрешенных зон; наличие вблизи поверхности полупроводника пластически деформированной области с металлической проводимостью и т.д. [5,14].

По описанной методике были получены зависимости тока растекания в системе зонд-образец от силы прижима зонда к поверхности кремния разных марок (рис. 5,a), из которых на основании разработанной модели (1)-(6) получены зависимости удельного сопротивления образцов кремния разных марок от силы прижима зонда к поверхности образца (рис. 5, b) и зависимость пороговой силы прижима зонда от удельного сопротивления кремния (рис. 6).

\section{Результаты и их обсуждение}

Анализ экспериментальных результатов исследования влияния окружающей среды на определение удельного сопротивления образца КДБ-10 показал, что при
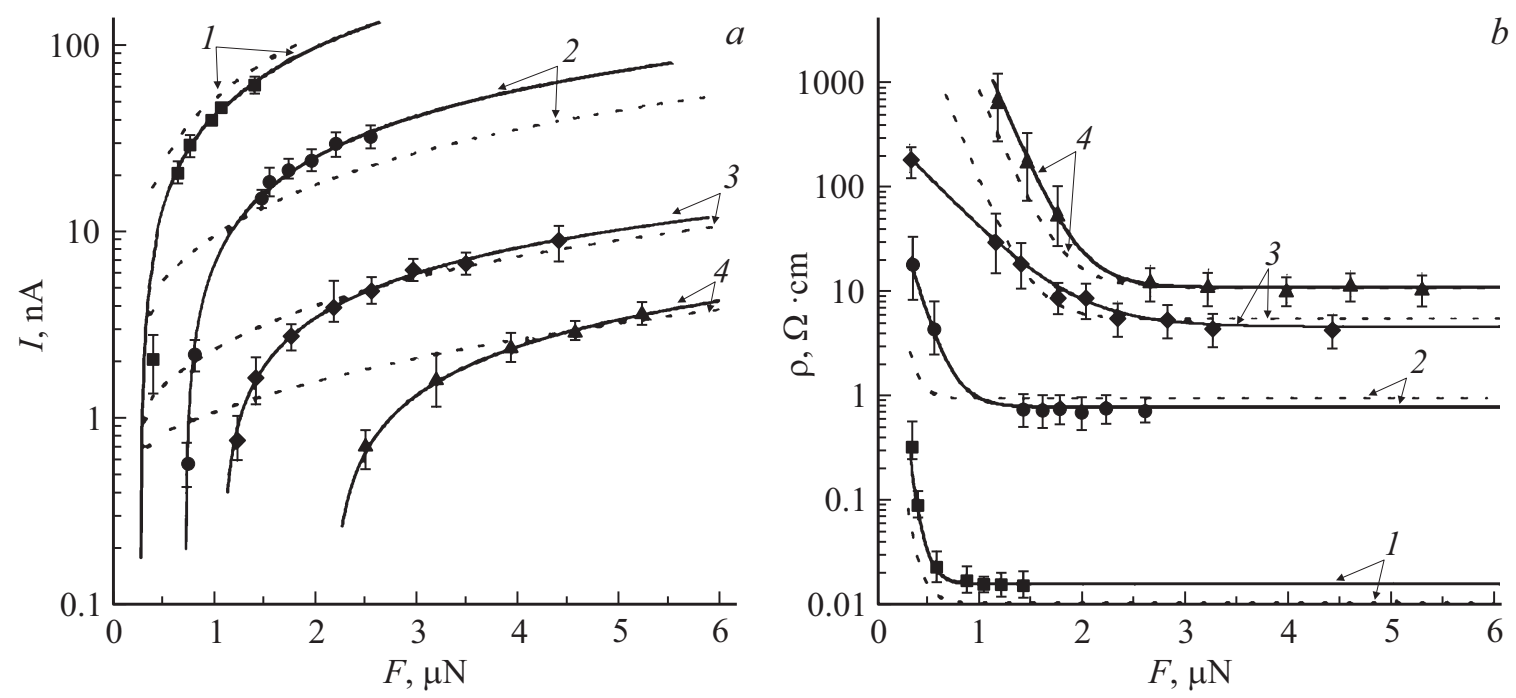

Рис. 5. Зависимости электрических параметров образцов кремния разных марок $(1-$ КЭС-0.01, $2-$ КДБ-1, $3-$ КДБ-5, 4 - КДБ-10) от силы прижима зонда к поверхности образца: $a-$ тока в системе зонд-образец, $b-$ удельного сопротивления. Штриховая линия - теоретическая зависимость (модель 1-6), сплошная линия - экспериментальная зависимость. 


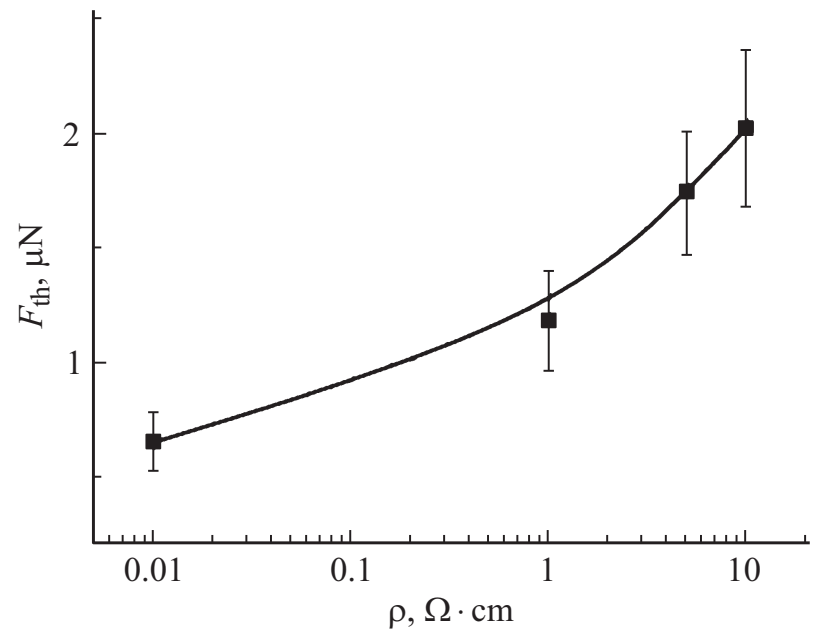

Рис. 6. Зависимость порогового значения силы прижима зонда к поверхности образца от удельного сопротивления кремния.

приложении напряжения к системе зонд-образец при атмосферном давлении происходит локальное анодное окисление поверхности подложки с участием адсорбированной пленки воды [15-18]. Анализ рис. 2, $a$ показал, что ток растекания по поверхности образца КДБ-10 в центральной области АСМ изображения составляет в среднем $0.1 \mathrm{nA}$, а по периферийной области - в среднем $0.6 \mathrm{nA}$. Полученный контраст распределения тока растекания соответствует процессу локального анодного окисления центральной области размером $5 \times 5 \mu$ m при первичном сканировании в условиях атмосферного давления. Исследование поверхности образца КДБ-10 при тех же режимах, но в условиях сверхвысокого вакуума $\left(10^{-8} \mathrm{~Pa}\right)$ показало, что в центральной области АСМ изображения ток растекания составляет в среднем $2 \mathrm{nA}$, а по периферийной области - в среднем $0.3 \mathrm{nA}$. Полученный контраст распределения тока растекания можно объяснить процессом удаления природного слоя окисла при сканировании центральной области размером $5 \times 5 \mu \mathrm{m}$ в контактном режиме АСМ в условиях сверхвысокого вакуума.

На основе полученных экспериментальных значений токов растекания в системе зонд-образец и разработанной математической модели (1)-(6) были определены значения удельного сопротивления образца КДБ-10, которые для исследований при атмосферном давлении составили $166 \Omega \cdot \mathrm{cm}$, а в условиях сверхвысокого вакуума $-10 \Omega \cdot \mathrm{cm}$, что соответствует спецификации (см. таблицу).

Таким образом, показано, что для получения достоверных данных об электрофизических параметрах полупроводниковых материалов и структур методом АСМ ОСР необходимо проводить исследования в условиях сверхвысокого вакуума для предотвращения процесса локально-анодного окисления поверхности исследуемого образца.
Анализ экспериментальных результатов по исследованию влияния силы прижима зонда к поверхности образца КЭС-0.01 на ток в системе зонд-подложка методом АСМ ОСР (рис. 3) показал, что при силе прижима $2 \mu \mathrm{N}$ наблюдается формирование стабильного контакта зонд-образец (рис. 3,c), что подтверждается при исследовании остальных образцов кремния (см. таблицу). Таким образом, следует выделить три области режимов исследований полупроводниковых образцов методом АСМ ОСР в зависимости от силы прижима зонда к поверхности образца.

1.При малых нагрузках (для КЭС-0.01 менее $1 \mu \mathrm{N}$ ) зонд слабо взаимодействует с поверхностью. Проведение исследований полупроводников методом АСМ ОСР в этом режиме нецелесообразно.

2. При средних нагрузках (для КЭС-0.01 более $1 \mu \mathrm{N}$ и менее $2 \mu \mathrm{N}$ ) зонд взаимодействует с поверхностью, в этом режиме методом АСМ ОСР можно проводить исследования по выявлению артефактов с сопротивлением, отличным от сопротивления образца.

3. При больших нагрузках (для КЭС-0.01 более $2 \mu \mathrm{N}$ ) наблюдается стабильный контакт зонда с поверхностью образца. При этом удаляются поверхностные артефакты и слои природного окисла.

Результаты исследований влияния силы прижима зонда на ВАХ образцов КЭС-0.01 и КДБ-1 показаны на рис. 4. Анализ представленных зависимостей и соотношений (1)-(6) показывает, что возрастание тока в системе зонд-образец при увеличении силы прижима зонда к поверхности образца связано прежде всего с уменьшением сопротивления контакта острия зонда с поверхностью образца и увеличением площади контакта зонд-образец.

Показана хорошая корреляция экспериментальных и теоретических результатов исследований влияния силы прижима зонда к поверхности кремния различных марок на значения токов в системе зонд-образец (рис. 5, $a$ ), особенно при больших силах прижима, что подтверждает адекватность разработанной математической модели определения удельного сопротивления полупроводников материалов. Анализ зависимостей удельного сопротивления кремниевых подложек различных марок от силы прижима зонда к поверхности образца (рис. $5, b$ ) показал наличие порогового значения силы прижима, при превышении которого удельное сопротивление исследуемого методом АСМ ОСР полупроводникового материала определяется наиболее достоверно. Анализ зависимости порогового значения силы прижима зонда к поверхности образца от удельного сопротивления кремния (рис. 6) показал, что при увеличении удельного сопротивления кремния пороговое значение силы прижима зонд-образец увеличивается, что может быть связано с эффектами, входящими в корректирующий фактор CF, а также с необходимостью удаления или обеспечения пробоя природного окисла кремния. 


\section{Заключение}

В работе представлены результаты экспериментальных и теоретических исследований определения электрических параметров подложек кремния различных марок методом АCM в режиме отображения сопротивления растекания. Исследовано влияние окружающей среды на значения токов растекания в системе зондподложка.

Показано, что исследование поверхности образцов кремния методом АСМ ОСР при атмосферном давлении приводит к снижению тока в системе зонд-образец, что связано с образованием пленки оксида кремния в ходе электрохимической реакции в адсорбированной на поверхности подложек пленке воды при приложении к системе зонд-подложка напряжения смещения. При проведении измерений в условиях сверхвысокого вакуума пленка оксида кремния не образуется, и результаты определения удельного сопротивления подложек кремния соответствуют спецификации образцов. Таким образом, для получения достоверных результатов исследования электрических параметров полупроводниковых материалов методом АСМ ОСР необходимо проводить измерения в условиях сверхвысокого вакуума.

Также в работе представлены результаты экспериментальных исследований влияния силы прижима зонда к поверхности подложки кремния марки КЭС-0.01 на ток в системе зонд-образец. Показано, что для определения удельного сопротивления необходимо формирование стабильного контакта зонд-образец.

Разработана математическая модель определения удельного сопротивления полупроводниковых материалов по значению тока растекания, определенного методом АСМ ОСР.

Показано, что возрастание тока в системе зондобразец при увеличении силы прижима зонда к поверхности образца связано с уменьшением сопротивления контакта острия зонда к поверхности образца и увеличением площади контакта зонд-образец.

Показана хорошая корреляция между экспериментальными и теоретическими результатами определения зависимостей влияния силы прижима зонда к поверхности образца на значения токов в системе зонд-образец для разных марок кремния.

Анализ зависимостей удельного сопротивления кремниевых подложек разных марок от силы прижима зонда к поверхности образца показал наличие порогового значения силы прижима, при превышении которого удельное сопротивление исследуемого полупроводникового материала определяется наиболее достоверно.

Полученные результаты могут быть использованы для разработки методик зондовой нанодиагностики и для исследования структур нано- и микроэлектроники, нанои микросистемной техники методом АСМ.

Работа выполнена при финансовой поддержке РФФИ (проекты № 16-29-14023 офи_м, 16-32-0069 мол_а) и
Южного федерального университета (проекты № ВнГр07/2017-02, № ВнГр-07/2017-26).

Результаты получены с использованием оборудования Научно-образовательного центра „Нанотехнологии“ Южного федерального университета (Таганрог).

\section{Список литературы}

[1] Hauquier F., Alamarguy D., Viel P. // Appl. Surf. Sci. 2012. Vol. 258. P. 2920-2926.

[2] Ghanem T.K., Williams E.D., Fuhrer M.S. // J. Appl. Phys. 2011. Vol. 110. P. 054305.

[3] Beinik I., Kratzer M., Wachauer A. // J. Appl. Phys. 2011. Vol. 110. P. 052005.

[4] Eyben P., Vandervorst W., Alvarez M. // Springer Handbook of Nanotechnology. 2007. P. 31-49.

[5] Clarysse T., Vanhaeren D., Hoflijk I., Vandervorst W. // Mater. Sci. Engineer. 2004. P. 123-206.

[6] Alekseev A., Chen D., Ghislandi M.G., De With G., Tkalya E.E., Syurik Y., Ageev O., Loos J. // Advanced Functional Materials. 2012. Vol. 22. N 6. P. 1311-1318.

[7] Syurik J., Alyabyeva N., Ageev O.A., Alekseev A. // Composites Sci. and Technol. 2014. Vol. 95. P. 38-43.

[8] Syurik J., Ageev O.A., Cherednichenko D.I., Konoplev B.G., Alexeev A. // Carbon. 2013. Vol. 63. P. 317-323.

[9] Ageev O.A., Konoplev B.G., Rubashkina M.V. // Nanotechnologies in Russia. 2013. Vol. 8. P. 23-28.

[10] Ageev O.A., Smirnov V.A., Fedotov A.A. // Nanotechnologies in Russia. 2012. Vol. 7. P. 47-53.

[11] Ageev O.A., Smirnov V.A., Solodovnik M.S. // Semiconductors. 2012. Vol. 46. N 13. P. 1616-1621.

[12] Kalinin S., Gruverman A. Electrical and Electromechanical Phenomena at the Nanoscale // Springer Handbook of Nanotechnology. 2007. P. 31-49.

[13] Frammelsberger W., Benstetter G., Kiely J., Stamp R.// Appl. Surf. Sci. 2007. Vol. 253. P. 3615-3626.

[14] Tanuma S., Powell C.J., Penn D.R. // Surf. Interface Analysis. 1988. Vol. 11. P. 577-589.

[15] Ageev O.A., Alyab'eva N.I., Konoplev B.G. // Semiconductors. 2010. Vol. 44. N 13. P. 1703-1708.

[16] Avilov V.I., Ageev O.A., Smirnov V.A., Tsukanova O.G. // Semiconductors. 2014. Vol. 48. N 13. P. 1757-1762.

[17] Avilov V.I., Ageev O.A., Smirnov V.A., Solodovnik M.S., Tsukanova O.G. // Nanotechnologies in Russia. 2015. Vol. 10. N 3. P. 214-219.

[18] Avilov V.I., Ageev O.A., Blinov Y.F., Konoplev B.G., Polyakov V.V., Smirnov V.A., Tsukanova O.G. // Technical Phys. 2015. Vol. 60. N 5. P. 717-723. 